\title{
Field Experiments of a Surface-Penetrating Radar for Mars
}

\author{
C. Leuschen*, P. Kanagaratnam, K. Yoshikawa, \\ S. Arcone, and P. Gogineni \\ *The Johns Hopkins University, Applied Physics Laboratory \\ 11100 Johns Hopkins Road, Laurel, MD 20723 \\ Phone: (443) 778-7662, Fax: (443) 778-5548 \\ E-mail: Carl.Leuschen@jhuapl.edu
}

\begin{abstract}
Using ground-penetrating radars to investigate the subsurface of Mars will be a key scientific objective over the next several years, especially in light of the large possibility that water could exist within the planet. Radars operating from a few megahertz up to a gigahertz will be able to provide valuable information concerning the subsurface electrical structure at resolutions ranging from a few centimeters near the surface to a few tens of meters at greater depths. One of the major goals of the work presented was to develop a lightweight, low-power, frequency-modulated radar system that could be used to detect subsurface deposits of ice and water. An inexpensive prototype system was developed using off-the-shelf connectorized components and evaluation boards. To verify the operation of this prototype system, a preliminary experiment was conducted in Lawrence, Kansas. Next, experiments were conducted over locations containing permafrost and ice in Fairbanks, Alaska. Results from these experiments are presented.
\end{abstract}

\section{INTRODUCTION}

For the preliminary experiments and testing, a simple surface-penetrating radar system was constructed [1]. The purpose of the radar system is to provide a test-bed to investigate radar performance, system trade-offs, signal processing, data interpretation, and antenna subsystems. The prototype was built with inexpensive evaluation boards and connectorized components. The system uses a direct digital synthesizer and can generate a variety of waveforms including a simple pulse, chirped pulse, FMCW or other modulated signal. For the initial testing presented in this paper, a chirped pulse waveform was used to achieve low peak power, high average power, and fine resolution. This paper begins by describing the basic construction and operation of the system. Next, the field tests are presented.

\section{RADAR SYSTEM}

\section{A. Transmitter and Receiver}

The prototype transmitter/receiver subsystem block diagram is shown in Figure 1, and the specifications are listed in Table 1. The transmitter subsystem generates a $5 \mathrm{MHz}$ to $120 \mathrm{MHz}$ chirp signal after receiving a trigger from the system controller (laptop). The transmitter is capable of generating frequencies down to D.C., but $5 \mathrm{MHz}$ is the lower bound of the transmit antenna. The chirp signal is generated using a $300 \mathrm{MHz}$ direct digital synthesizer (DDS) evaluation board. The signal is amplified with a high-power amplifier. With a power divider, the amplified signal is split into two parts, one for the transmit antenna subsystem and the other to serve as a local oscillator for the receiver mixer that is used to de-chirp the received signal.

The receiver subsystem down converts the input RF response and digitizes the resulting waveform. The signal is down converted by mixing a replica of the transmit signal with the receive signal producing a collection of beat frequency signals. These signals are low-pass filtered to remove the higher frequency components generated by the mixer. After low-pass filtering, a single-pole high-pass filter provides attenuation of the direct antenna coupling and an increased gain for the weak subsurface returns. This highpass filter is analogous to range gating or time controlled gain in an "impulse"-type time-domain radar system. The highpass output is amplified and then digitized by a 2.5 MSPS 16bit analog-to-digital converter evaluation board.

All timing and control of the system are accomplished through the parallel port of a laptop computer and with a few low-speed digital integrated circuits. Evaluation software was used to trigger the system and store the data to memory. The complete system was constructed for less than a thousand dollars. Excluding batteries and the antenna subsystems, the system weighs about $1.5 \mathrm{~kg}$ with dimensions of $7 \mathrm{~cm} \times 20 \mathrm{~cm} \times 30 \mathrm{~cm}$. The complete system could be easily miniaturized using a single printed circuit board and surface mount components to easily satisfy the weight and volume constraints of a rover.

\section{B. Antenna Subsystems}

For initial testing with the radar system, two bowtie antennas were constructed to operate over the frequency range $10 \mathrm{MHz}$ to $120 \mathrm{MHz}$. Due to the large size required to radiate at the lower frequencies, the antennas needed to be collapsible for storage and transportation. An aluminum screen was used since it was lightweight, the fine mesh behaved as a solid conductor over the frequency range of interest, and it could be easily stored for transportation and deployment in the field. A final design was selected using two $1.3 \mathrm{~m}$ equilateral triangles supported by a PVC frame. Preliminary testing of the antennas over dry sand $\left(\varepsilon_{r}=2.5\right)$ with a network analyzer and 4:1 balun transformer showed a $3 \mathrm{~dB}$ lower cutoff at about $20 \mathrm{MHz}$ with a resistive impedance around $50 \Omega$ at the input of the transformer (200 $\Omega$ input resistance for the antenna [2]). 
TABLE I

SYSTEM SPECIFICATIONS

\begin{tabular}{|l|l|}
\hline Modulation & Swept-FM, Stepped-FM, Pulsed \\
\hline Frequency & Programmable up to $120 \mathrm{MHz}$ \\
\hline Sweep Time (Pulse Width) & Programmable \\
\hline Power Output & $10 \mathrm{dBm}$ \\
\hline Total Power Consumption & $<3 \mathrm{Watts}$ \\
\hline ADC Resolution & $16 \mathrm{bits}$ \\
\hline ADC Dynamic Range & $96 \mathrm{~dB}$ (theoretical) \\
\hline ADC Sampling Rate & $2.5 \mathrm{MSPS}$ \\
\hline Size & $7 \mathrm{~cm} 20 \mathrm{~cm} \times 30 \mathrm{~cm}$ \\
\hline Weight & $1.5 \mathrm{~kg}$ \\
\hline
\end{tabular}

A different concept was considered for the receiving antenna. There is a slight difference in the specific roles of transmit and receive antennas concerning power transfer. The transmit antenna must radiate power efficiently into the ground, whereas the receive antenna only needs to measure the incident electric field. Due to this difference, matching the receive antenna for maximum power transfer is not a concern as long as the electric field is being measured. For a receiving antenna, the effective length is equal to the voltage induced upon the terminals when the antenna is open circuited. For the case of an electrically small dipole, the effective length is approximately equal to half of the physical length. More importantly, if the voltage induced upon the antenna terminals can be measured while the antenna is open circuited, an electrically small antenna could be used as the receive antenna. This can be accomplished using a high input-impedance, high-bandwidth, operational amplifier at the antenna terminals.

\section{Complete System}

Figure 2 shows the complete surface-penetrating radar. All the systems, including the transmitter, receiver, antennas, batteries, and laptop, are mounted on a wooden ski/sled that can be dragged as data are being collected. The bowtie antenna is located at the rear of the ski, and the transmitter is placed near to the bowtie antenna. The receive antenna is placed as far as possible on the ski from the bowtie to reduce the antenna feed-through signal. The transmitter/receiver box, laptop computer, and batteries are located between the antennas on the ski. The entire system is portable and requires no external power or control cables.

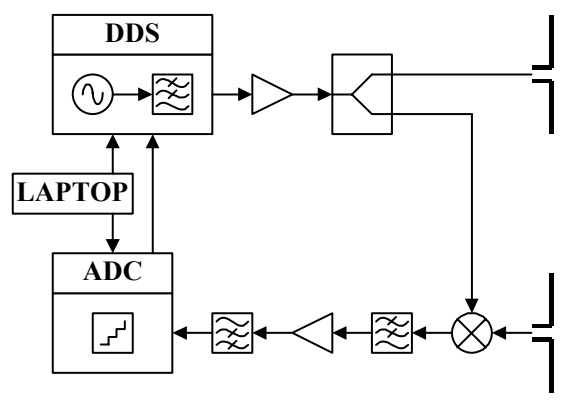

Figure 1. Radar block diagram.

\section{FIELD EXPERIMENTS}

To evaluate the performance of the prototype system, measurements were collected over a few locations including one site in Lawrence, Kansas, and others near Fairbanks, Alaska. For most of these experiments, data were collected over stratigraphy that was, to some extent, already determined through seismic surveys, dilled cores, or other geological surveys. This section begins with description of the preliminary experiment in Lawrence, Kansas. Next, the results from an experiment in Alaska is presented.

\section{A. Lawrence, Kansas}

The first experiment was conducted outside Moore Hall (Kansas Geological Survey) in the west campus of the University of Kansas. The site was selected because, previous to the radar measurements, a seismic survey was conducted by the Kansas Geological Survey [3]. The radar measurements were collected over a traverse of approximately $30 \mathrm{~m}$ with a sample interval of about $0.33 \mathrm{~m}$ between traces. The raw data are displayed in Figure 3a. The vertical axis of this figure indicates the instantaneous transmit frequency as the radar sweeps from $5 \mathrm{MHz}$ to $120 \mathrm{MHz}$. The dominating sinusoidal response is the beat frequency associated with the antenna feed through. Two important features can be noticed in the unprocessed data. First, there exists significant transmitted and received power over the entire frequency range of $5 \mathrm{MHz}$ to $120 \mathrm{MHz}$. This indicates that the system is efficiently operating over more than four octaves of the frequency spectrum. Second, two external noise sources are detected at $91.5 \mathrm{MHz}$ and $30.5 \mathrm{MHz}$, as shown by the horizontal lines in the amplitude plot on the right of Figure 3a. This external noise is attributed to a local FM radio station, KANU, at $91.5 \mathrm{MHz}$. The component at $30.5 \mathrm{MHz}$ is a non-linear effect of the receive mixer.

The radar profile is shown in Figure 3b. This time-domain response is obtained by evaluating the beat frequency spectrum of the collected data via an inverse Fourier transform. A Hanning window [4] is applied before the IFFT is performed. Finally, a gain function is applied to the data to amplify the deeper reflection.

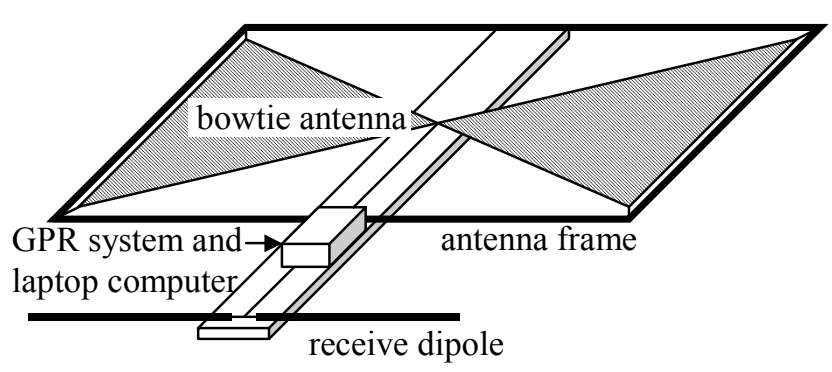

Figure 2. Complete radar system. 

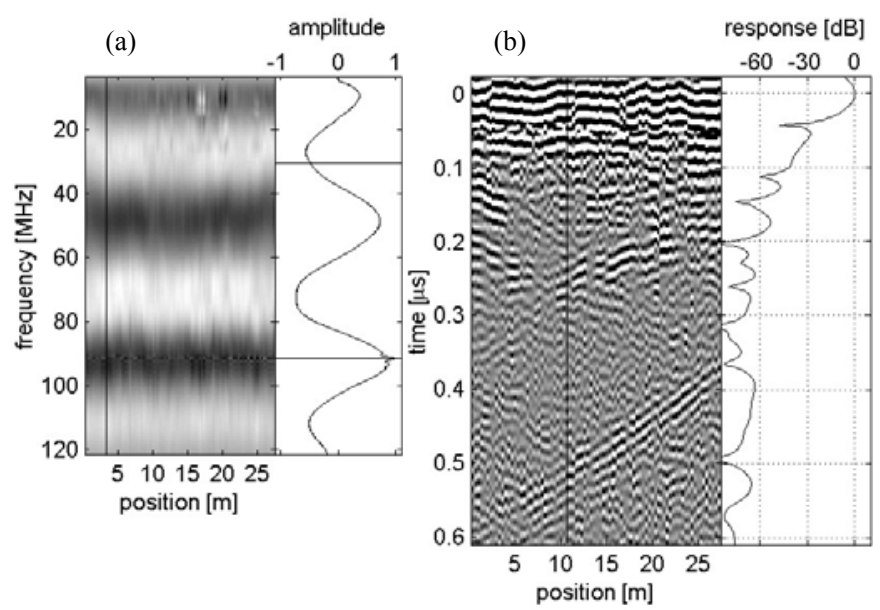

Figure 3. Raw data and radar profile for the Kansas site.

\section{B. Fairbanks, Alaska}

From August $19^{\text {th }}$ to August $31^{\text {st }}$ of 2001, experiments were conducted around Fairbanks, Alaska. The major goals were to verify the performance of the system and to investigate the use of surface-penetrating radar to detect permafrost and the presence of water or ice within permafrost. Unfortunately, due to the time of year, a significant amount of rain fell before and during the experiments. Due to this large amount of precipitation, the upper meter or so of the test sites (known as the "active layer") was saturated with water.

One field experiment in Alaska was conducted at Fort Wainwright near Fairbanks. At the site, a 4-ft-wide line was cut through a spruce forest and was approximately $300 \mathrm{~m}$ in length before it intersected a small pond resulting from recent flooding. From previous radar surveys and drilling, the subsurface is thought to consist of a near-surface saturated thaw layer overlying discontinuous permafrost. Beneath the permafrost could lie a small layer of wet soil above the water table and bedrock.

Data were collected continuously along the $300 \mathrm{~m}$ traverse with the prototype system. The spacing was approximately 3 $\mathrm{cm}$ per trace for a total of 10000 traces. Several events can be detected in the image as shown in Figure 4. The first is the continuous reflection ranging from about 300 ns to 350 ns. This reflection ranges from $0 \mathrm{~m}$ to $50 \mathrm{~m}$ at $300 \mathrm{~ns}$, it slowly decrease from $50 \mathrm{~m}$ to $70 \mathrm{~m}$, and then extends from 70 $\mathrm{m}$ to $150 \mathrm{~m}$ at about $350 \mathrm{~ns}$. This layer is picked up again at around $190 \mathrm{~m}$ and is seen off and on to the end of the traverse. This reflection may indicate the transition from the frozen permafrost to a wet soil. Beneath this reflection at about $410 \mathrm{~ns}$ is a relatively flat layer extending from $0 \mathrm{~m}$ to $80 \mathrm{~m}$. There is also a weaker reflection around $400 \mathrm{~ns}$ from $100 \mathrm{~m}$ to $150 \mathrm{~m}$. There seems to be many near-surface events within the image. The most prominent shown across the entire image is the ringing of the near-surface active thaw layer.

\section{CONCLUSIONS}

Based on the simulations and experiment results, an FM$\mathrm{CW}$ system operating between a few megahertz to a few hundreds of megahertz is proposed for a lander/rover-based mission. The radar developed for this research operated from $5 \mathrm{MHz}$ (constrained by the lower limit of the transmit antenna) to $120 \mathrm{MHz}$ (constrained by the upper limit of the direct digital converter not the antenna subsystems).

\section{REFERENCES}

[1] Leuschen, C., Surface-Penetrating Radar for Mars Exploration, Dissertation, The University of Kansas, 2001.

[2] Compton, R. C., R. C. McPhedran, Z. Popovic, G. M. Gebeiz, P. P. Tong, and D. B. Rutledge, "Bow-tie antennas on a dielectric half-space: theory and experiment," IEEE Transactions on Antennas and Propagation, Vol. 35, pp. 622-631, 1987.

[3] Knapp, R.W., "Vertical resolution of thick beds, thin beds, and thin bed cyclotherms," Geophysics, Vol. 55, pp. 1183-1190, 1990.

[4] Harris, F. J., "On the use of windows for harmonic analysis with the discrete Fourier Transform," Proceedings of the IEEE, Vol. 66, pp. 51-83, 1978.

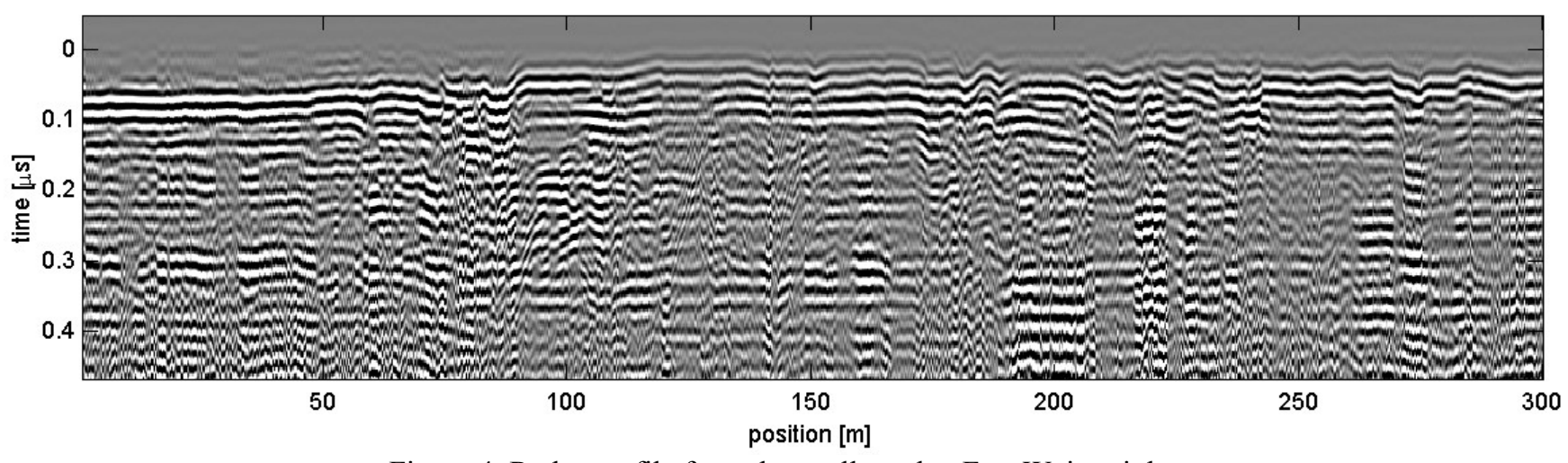

Figure 4. Radar profile from data collected at Fort Wainwright. 